\title{
IMPACTO DE LA PUBLICIDAD DE MARCA EN DIFERENTES MEDIOS DE COMUNICACIÓN MASIVA
}

\author{
María Cristina Otero Gómez* \\ Wilson Giraldo Pérez** \\ Ivan Darío Giraldo Pérez*** \\ DOI: https://doi.org/10.33571/revistaluciernaga.v11n21a8
}

\section{Resumen}

La investigación de la que surgió este artículo tuvo como objetivo analizar el impacto del uso de diferentes medios tradicionales de comunicación masiva y su relación con la fortaleza de marca en el segmento juvenil. Se utilizó el modelo BrandZ de la consultora Millward Brown. Se seleccionaron jóvenes universitarios de ambos sexos, con edades entre 18 y 24 años para conformar la muestra con 60 personas. Se observó que la combinación de los medios televisión + radio + revistas es la que genera mayor crecimiento en la variable vínculo emocional, pero no es la mejor opción de inversión en comunicación publicitaria. Por esta razón las empresas implementan diversas combinaciones dependiendo del ciclo de vida del producto. Esto con el fin de inducir al consumidor al uso de un bien o un servicio mediante la relación necesidad-satisfacción con la marca, que presentan los medios de comunicación con fines publicitarios.

Palabras clave: comunicación; marca; publicidad; consumidor.

Recibido. Marzo 26, 2019 Aceptado. Marzo 30, 2019

\footnotetext{
* Profesional en Comercio Internacional, Magíster en Mercadeo, Directora del Grupo de Investigación Dinámicas de Consumo de la Facultad de Ciencias Económicas de la Universidad de los Llanos. Villavicencio - Colombia. Orcid: http://orcid.org/0000-0002-3241-7877; e-mail: motero@unillanos.edu.co

** Administrador de Empresas, Magíster en Mercadeo, docente de la Facultad de Ciencias Económicas de la Universidad de los Ilanos. Villavicencio - Colombia. Orcid: http://orcid.org/0000-0001-8514-9805; e-mail: wgiraldo@unillanos.edu.co

*** Profesional en Mercadeo y Publicidad; Especialista en Gerencia de publicidad; Especialista en Comunicación Corporativa. Marketing Science Director OMD. Bogotá - Colombia. e-mail: igiraldo@omd.com.co
} 


\title{
THE IMPACT OF BRAND ADVERTISING ON DIFFERENT MASS MEDIA
}

\author{
María Cristina Otero Gómez* \\ Wilson Giraldo Pérez** \\ Ivan Darío Giraldo Pérez*** \\ DOI: https://doi.org/10.33571/revistaluciernaga.v11n21a8
}

\section{Summary}

This article is the result of a research that aimed at analyzing the impact of different traditional mass media and their relationship with brand strength in the youth segment. The BrandZ model of the consultancy firm Millward Brown was used. Young university students of both sexes, aged between 18 and 24, were selected to make up the sample of 60 people. It was observed the mixed media of television + radio + magazines is the one that generates the greatest growth in the emotional bond variable, but it is not the best option for investment in advertising communication. For this reason, companies implement different combinations depending on the life cycle of the product, in order to induce the consumer to use a good or a service through the relationship need-satisfaction and brand, presented by the media for advertising purposes.

Key words: communication; brand; advertising; consumer.

Received. March 26, 2019

Accepted. March 30, 2019

\footnotetext{
* Profesional en Comercio Internacional, Magíster en Mercadeo, Directora del Grupo de Investigación Dinámicas de Consumo de la Facultad de Ciencias Económicas de la Universidad de los Llanos. Villavicencio - Colombia. Orcid: http://orcid.org/0000-0002-3241-7877; e-mail: motero@unillanos.edu.co

** Administrador de Empresas, Magíster en Mercadeo, docente de la Facultad de Ciencias Económicas de la Universidad de los Ilanos. Villavicencio - Colombia. Orcid: http://orcid.org/0000-0001-8514-9805; e-mail: wgiraldo@unillanos.edu.co

*** Profesional en Mercadeo y Publicidad; Especialista en Gerencia de publicidad; Especialista en Comunicación Corporativa. Marketing Science Director OMD. Bogotá - Colombia. e-mail: igiraldo@omd.com.co
} 


\title{
IMPACTO DA PUBLICIDADE DA MARCA EM DIFERENTES MEIOS DE COMUNICAÇÃO SOCIAL
}

\author{
María Cristina Otero Gómez* \\ Wilson Giraldo Pérez** \\ Ivan Darío Giraldo Pérez*** \\ DOI: https://doi.org/10.33571/revistaluciernaga.v11n21a8
}

\section{Resumo}

A pesquisa que levou a este artigo teve como objetivo analisar o impacto do uso de diferentes meios de comunicação de massa tradicionais e sua relação com a força da marca no segmento jovem. Foi utilizado o modelo BrandZ da empresa de consultoria Millward Brown. Foram selecionados jovens universitários de ambos os sexos, entre 18 e 24 anos, para compor a amostra de 60 pessoas. Observou-se que a combinação de televisão + rádio + revistas é a que gera maior crescimento na variável vínculo emocional, mas não é a melhor opção para investimento em comunicação publicitária. Por este motivo, as empresas implementam diferentes combinações dependendo do ciclo de vida do produto. Isto para induzir o consumidor a utilizar um bem ou serviço através da relação necessidade-satisfação com a marca, apresentada pela mídia para fins publicitários.

Palavras-chave: comunicação; marca; publicidade; consumidor.

Recebido. Março 26, 2019 Aceitado. Março 30, 2019

\footnotetext{
* Profesional en Comercio Internacional, Magíster en Mercadeo, Directora del Grupo de Investigación Dinámicas de Consumo de la Facultad de Ciencias Económicas de la Universidad de los Llanos. Villavicencio - Colombia. Orcid: http://orcid.org/0000-0002-3241-7877; e-mail: motero@unillanos.edu.co

** Administrador de Empresas, Magíster en Mercadeo, docente de la Facultad de Ciencias Económicas de la Universidad de los Ilanos. Villavicencio - Colombia. Orcid: http://orcid.org/0000-0001-8514-9805; e-mail: wgiraldo@unillanos.edu.co

*** Profesional en Mercadeo y Publicidad; Especialista en Gerencia de publicidad; Especialista en Comunicación Corporativa. Marketing Science Director OMD. Bogotá - Colombia. e-mail: igiraldo@omd.com.co
} 


\section{Introducción}

Las empresas compiten en un mercado donde existe una amplia y creciente oferta de productos que están contenidos en diversas categorías. Esta oferta, según el Departamento Administrativo Nacional de Estadística de Colombia (DANE, 2014), es definida como productos de la canasta familiar y se trata de los bienes y servicios que puede demandar cualquier hogar en el país, incluyendo a aquellos de ingresos bajos, medios o altos.

Por estas razones, las empresas se esfuerzan constantemente en el desarrollo de bienes y servicios orientados a satisfacer las necesidades y deseos del ser humano. Pero no basta con crearlos, es necesario dar a conocer y generar recordación de los productos en los consumidores. De allí que muchos empresarios se fijen como objetivo el posicionamiento de la marca, puesto que es el encargado de "crear percepciones mentales con respecto a la marca en la mente de los consumidores y, por lo tanto, ayuda al comercializador a vender la misma" (Lakshmi y Kavida, 2018, p.1). El término posicionamiento tiene diferentes interpretaciones. Según Fischer y Espejo (2011), el posicionamiento influye en la percepción mental que los consumidores tienen de una marca, producto, o empresa, en relación con la competencia; Keller (2003) lo asocia a la superioridad de una marca en la mente de los consumidores; y Wang (2017) advierte que es clave para la diferenciación de una marca frente a sus competidores.

Lo anterior refleja la ausencia de un consenso para definir el término posicionamiento, pero esto no significa que tampoco existan elementos afines que permitan alcanzar la recordación de la marca, la cual se logra mediante el desarrollo de una comunicación adecuada cuya finalidad es entregar los mensajes deseados, al público objetivo.

Uno de estos elementos afines, es la capacidad que tiene la publicidad en la construcción de marca, la cual se apoya en los medios de comunicación para que las organizaciones den a conocer y promocionen sus productos. Así lo sugieren Berger, Wagner y Schwand (2012) al afirmar que "los anunciantes pueden elegir estímulos altamente sobresalientes o emocionalmente excitantes que atraigan la atención visual de los consumidores" (p. 411), esto para hacer referencia a la televisión y medios impresos. En cuanto a la comunicación sonora, Kilian (2009) sostiene que en un contexto de saturación mediática el sonido -y más concretamente- la música, permiten a las marcas incrementar su valor y fidelidad entre su público, dotar de coherencia a sus mensajes y generar una experiencia inmersiva. Estos postulados conllevan a inferir que la publicidad es uno de los elementos presentes en el medio que puede modificar las preferencias del individuo.

Por ello, se reconoce la necesidad de evaluar la incidencia del uso de los diferentes medios publicitarios tradicionales, como generadores de recordación y con ello, de posicionamiento. Al respecto, Gelb, Pickett y Zhang (Citados por Cifuentes y Sánchez, 2006) afirman que, para lograr una actitud positiva hacia la marca, debe existir primero, una actitud positiva hacia el anuncio; por tanto, la publicidad cumple una función de puente entre la marca y el consumidor (López, 2010). 


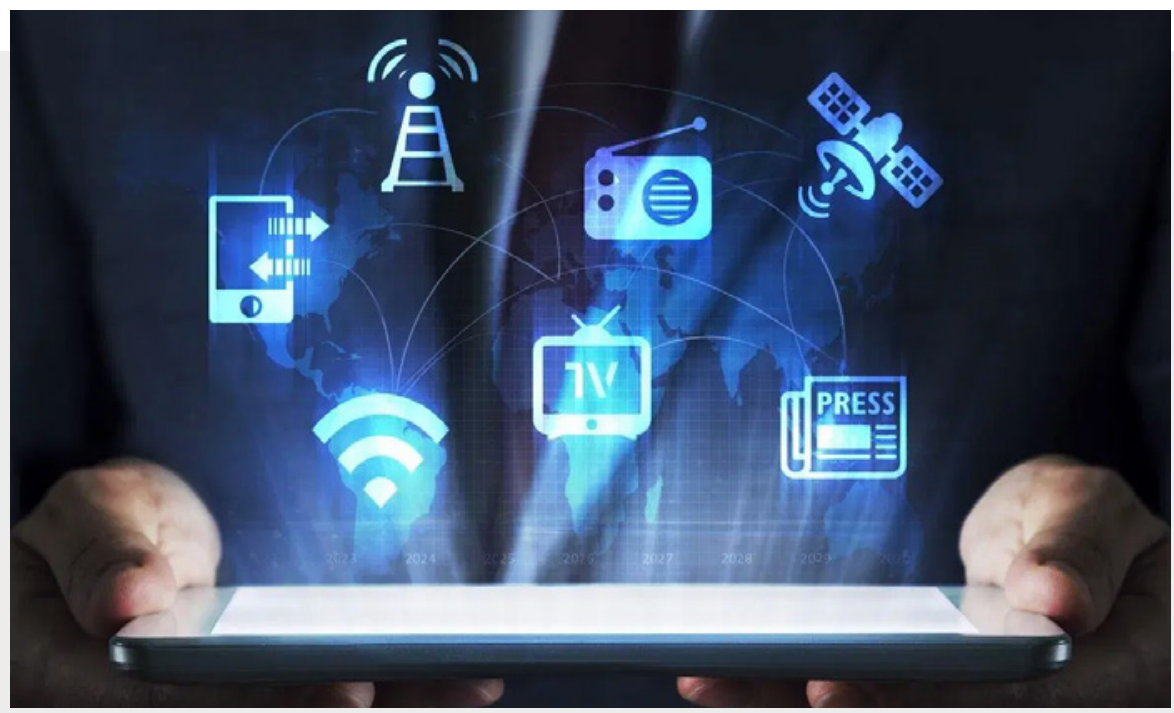

Imagen: https://bit.ly/2p0ozTP

En este sentido, es relevante la cuantificación de la influencia de los medios masivos y tradicionales de comunicación sobre la construcción de la fortaleza de marca. Para ello se ha tomado como población objeto de estudio al segmento juvenil, quienes actúan de forma diferente según el entorno social. Aunque en la cotidianidad los jóvenes demuestran un alto uso de las Tecnologías de la Información y la Comunicación (TIC), también hacen uso de los medios tradicionales de comunicación. Según la Encuesta sobre Consumo Cultural, durante el año 2017 de un total de 28.023 personas que vieron televisión el 30\% corresponde a jóvenes; asimismo, de 19.266 personas que escucharon radio, el $24 \%$ corresponde a la población joven (DANE, 2018).

Estos datos se convierten en una oportunidad, puesto que según la misma encuesta los jóvenes son el segundo grupo etario que más consume contenido televisivo y el tercer grupo a nivel de consumo radial. Aunque la encuesta no revela información para los medios impresos, resulta conveniente indagar sobre el desempeño de estas fuentes o la combinación de estas y su aporte a la construcción de la fortaleza de la marca.

Este estudio se ha propuesto como objetivo analizar el impacto del uso de diferentes medios tradicionales de comunicación masiva y su relación con la fortaleza de marca en el segmento juvenil. En particular, el producto objeto de análisis es el polvo efervescente Bonfiest Lua desarrollado por la firma Tecnoquímicas. Se ha tomado como referencia un estudio realizado en Estados Unidos donde se revela que la publicidad:

"para medicamentos se transmite a través de medios tradicionales como la televisión, la radio, las revistas, los periódicos y las vallas publicitarias, solo se dedica una pequeña pero creciente porción a mostrar anuncios (banners) y búsquedas patrocinadas en Internet"

(Chesnes y Jin, 2019, p.2).

Debido a la ausencia de estudios en Colombia que revelen la efectividad de la publicidad de los medios masivos tradicionales de comunicación en tiempos actuales, proponemos este estudio con el fin de enriquecer la literatura relacionada con los procesos de construcción y gestión de la marca. 
En razón a que Tecnoquímicas es una de las compañías más fuertes en el mercado farmacéutico colombiano, en el avance de la presente investigación también se considera la literatura empresarial, junto con la literatura científica. Desde esta perspectiva se asume la propuesta de medida de carácter comercial "BrandZ" desarrollada por la firma consultora Millward Brown, la cual, a partir de un modelo gráfico presenta un enfoque jerárquico que determina la fortaleza de la relación que establece una marca con el consumidor.

El presente estudio [1] busca trascender en la interpretación expuesta en la literatura sobre las Comunicaciones Integradas de Marketing (CIM), pues en diferentes escritos, el análisis se ha centrado, principalmente, en demostrar las ventajas económicas y financieras que su uso genera para la empresa (Schultz y Kitchen, 2000), pero poco se ha avanzado en la investigación sobre los efectos de la integración de las herramientas de comunicación disponibles, particularmente en Colombia.

A partir de lo anterior, se formularon las siguientes preguntas de investigación ¿Cuál es la combinación de medios tradicionales de comunicación masiva más efectiva, que permite alcanzar niveles superiores de fortaleza de marca en el segmento juvenil? y ¿Cuál es el medio de comunicación tradicional masivo capaz de mejorar los indicadores de fortaleza de marca en el segmento juvenil?

Para dar respuesta a este interrogante y al objetivo planteado, el trabajo se organiza a partir de una revisión de literatura, seguido de los métodos empleados para analizar la relación entre las variables de evaluación. Finalmente se discuten los resultados, seguidos por las conclusiones, así como las implicaciones prácticas y el planteamiento de nuevas temáticas de estudio.

\section{La publicidad, los medios de comunicación y la marca}

La publicidad es una forma de comunicación que se manifiesta en diferentes ámbitos de la sociedad, y sobre ella existen diversas posiciones, algunas a favor otras en contra. Por ejemplo, se ha cuestionado sobre el contenido de sus mensajes (Cortés, Marván y Lama, 2004); las técnicas implementadas (Ramírez, 2001); el poder influenciador sobre el consumidor (Golovina, 2014); y otros consideran que estigmatiza determinados segmentos del público (Uribe, Manzur, Hidalgo y Fernández, 2008). No obstante, resulta conveniente mencionar que en 1986 la Organización de las Naciones Unidas para la Educación, la Ciencia y la Cultura se pronunció refiriéndose a que los aspectos de la publicidad y la información son importantes en todas las sociedades. De ello se desprende que la publicidad "abarca todas las formas en que los individuos y las organizaciones anuncian las mercancías y los servicios que ofrecen y promueven una imagen positiva de ellos mismos" (Unesco, 1986, p.11).

Posteriormente, surge otro concepto donde la publicidad supone un proceso de comunicación entre un emisor y su mercado, con la capacidad de influir en determinado comportamiento; de este modo, Richards y Curran (2002) sostienen que "la publicidad es una forma de comunicación pagada y mediada de una fuente identificable, diseñada para persuadir al receptor para que tome alguna decisión, ahora o en el futuro" (p. 74). Así también, otros autores ven en la publicidad aspectos favorables como: 
Llegar a la gente inaccesible para los vendedores, dar a conocer los nuevos usos de un producto, introducir un nuevo producto, aumentar las ventas, ayudar a la fuerza de ventas. Para ello, la publicidad trata de modificar la disposición y el comportamiento de los posibles clientes, a través del suministro de información; modificando los deseos del consumidor y las preferencias de marca de los clientes (Degrado, 2005, p. 3).

En esta definición se destaca el suministro de información, el cual requiere de los medios de comunicación para llegar de forma adecuada a su grupo de interés. Al respecto Román (2000) menciona que "de forma pasiva o activa todos estamos ante los medios de comunicación. Nuestra existencia está condicionada y articulada por ellos" (p.128). Por esta razón en el contexto de la promoción y la publicidad se encuentra la presencia y operación de estas formas de comunicación. Entre sus principales funciones se encuentran "informar, entretener y ofrecer publicidad de productos y servicios" (García, 2003, p. 171). De allí que "los medios de comunicación tradicionales, lejos de desaparecer por el desarrollo tecnológico han ido avanzando y actualizándose" (Marín, 2006, p. 194). Tanto la televisión, como la prensa y la radio, se han reinventado mediante la implementación de nuevos formatos que permiten la formación de contenidos orientados a una audiencia específica. Por ejemplo, "cada vez más personas eligen ver películas y programas de televisión en Internet" (Wang, Zhuoa, Li, Ren y Zhang, 2018, p.1.)

Si bien es cierto que se ha afirmado que los medios de comunicación "poseen una enorme capacidad de influencia en el cambio y/o asentamiento de hábitos, patrones y conductas" (Calderón, 2014, p.6), también lo es, que no todos los medios tienen la misma habilidad para transmitir un mensaje que se vea reflejado en la captación de clientes para sus marcas. De allí la importancia en el análisis de la relación entre la escogencia del medio de comunicación y el fortalecimiento de la marca.

En coherencia con el planteamiento anterior, se demuestra que hay investigaciones orientadas a estudiar los efectos de la consistencia estratégica del mensaje en el consumidor. Dichos estudios infieren que, con mensajes de comunicación que transmiten información congruente en contenido y significado, se pueden producir efectos positivos en la construcción de marca. En esta línea, la tarea consiste en identificar los medios de comunicación en los cuales la empresa debe invertir para introducir un nuevo producto en el mercado, posicionarse de acuerdo con un determinado concepto, aumentar la recordación de la marca o motivar la compra del público objetivo (Torres y Muñoz, 2006).

Dependiendo del objetivo que pretende alcanzar cada organización existe una clasificación de medios indicados para lograrlo. Por ejemplo, Sanna (2013) considera que, si una organización busca un reconocimiento de marca "la televisión, las revistas, la publicidad en vía pública, internet y las redes sociales pueden ser apropiados, al igual que una comunicación de marketing directo" (p. 188). Asimismo, cuando se busca la recordación de marca "la televisión, la radio en todas sus formas, los periódicos y la red son medios apropiados para este objetivo. Las revistas tienen limitaciones en cuanto a su repetición" (p. 188).

Algunos estudios revelan que la escogencia de la marca, producto o empresa está influenciada por diferentes motivos, bien sea por moda, por estatus, por recomendación, por utilidad o por afinidad, entre otros. Por ejemplo, Golovina (2014) plantea que la comunicación masiva es un instrumento que moldea los 
motivos de compra; mientras que Rundle-Thiele y Bennett (2001) consideran que el comportamiento del consumidor juvenil varía en función del producto; y, Wood (2004) sostiene que la lealtad de los jóvenes en relación con una marca está determinada por la sensibilidad al precio.

En este sentido, otro factor que se debe considerar es el segmento al que está dirigida la publicidad de determinada marca, pues "los resultados muestran que la prensa y la radio son medios más distantes para los jóvenes, a diferencia de la televisión e Internet. Sin embargo, la credibilidad de la televisión es peor que la radio y la prensa" (Bernal, 2010, p. 1). A este respecto, Menéndez (2014) en su estudio sobre la presentación de los jóvenes en los medios de comunicación, específicamente en el caso de la prensa, la radio y la televisión, manifiesta que estos medios cada vez se encuentran más alejados de ellos y que sobresale la ausencia de contenidos dirigidos a la población joven. La misma autora sostiene que "los editores son conscientes de esta situación y de la necesidad de diseñar estrategias que les permitan mantener un target joven" (p.28). Por tanto, se convierte en un reto la identificación del mensaje y el medio o la combinación de los medios de comunicación tradicionales, adecuados para conectarse con el consumidor juvenil, con el fin de persuadirlo en el consumo de una marca.

\section{METODOLOGÍA}

Para medir la fuerza de la relación de la marca con el consumidor, se utilizó el Modelo BrandZ. Este modelo permite analizar, a partir de una escala jerárquica de 5 niveles, la lealtad que se tiene hacia la marca y los puntos en los cuales son fuertes o débiles estas relaciones (Brandz, 2013). De esta forma, se puede intervenir mediante acciones que apunten a contrarrestar las debilidades encontradas. Millward Brown sugiere que cada persona, en función de la Presencia (presence), la relevancia (relevance), el desempeño (performance), la ventaja (advantage) y el vínculo emocional (bonding) hacia una categoría o marca, se ubica en un nivel de la escala BrandZ. A partir de esto, se obtiene un perfil de relación del consumidor con las marcas, el cual soporta la preparación de planes y acciones de mercadeo, hasta poseer un conjunto de elementos que posibiliten su desempeño adecuado.

Para el desarrollo del estudio, se utilizó una metodología cuasiexperimental, trabajando con jóvenes universitarios de ambos sexos, con edades entre 18 y 24 años residentes en Bogotá, Colombia. Se conformó una muestra total de 6 grupos, cada uno con 10 integrantes, para un total de 60 personas. A cada una de ellas se le aplicó una prueba de entrada y otra de salida, orientadas a verificar la fortaleza de la marca Bonfiest dentro de productos de la misma categoría y también dentro de otras categorías de productos; todo esto a través de la exposición a diferentes medios de comunicación masiva. Como dice Segura (2003), los tipos de diseños cuasiexperimentales corresponden a estudios que establecen una medición previa a la intervención y otra posterior; además, "son factibles dado que se pueden realizar en pequeñas unidades, por lo cual son más baratos y tienen menos obstáculos prácticos" (p. 2). 
Los seis grupos se organizaron de la siguiente manera:

Grupo 1: Expuesto solamente a publicidad en televisión.

Grupo 2: Expuesto solamente a publicidad en radio.

Grupo 3: Expuesto solamente a publicidad en revistas

Grupo 4: Expuesto a combinación de publicidad en televisión y radio.

Grupo 5: Expuesto a combinación de publicidad en televisión y revistas.

Grupo 6: Expuesto a combinación de publicidad en televisión, radio y revistas.

La actividad se llevó a cabo durante varias jornadas académicas desarrolladas en condiciones normales de clase en dos universidades -una pública y otra privada-; como primera actividad de clase se aplicó una prueba de entrada que buscó determinar la fortaleza de la marca inicial a través del modelo BrandZ propuesto por la empresa Millward Brown. El siguiente paso consistió en aplicar un instrumento que reflejaba: la mención de la marca (presence); la recordación de exposición a la marca en medios de comunicación masiva (relevance); el lugar jerárquico que la marca ocupa en la mente del consumidor para determinada categoría, tanto de forma espontánea como asistida (performance); la marca favorita en la categoría estudiada (advantage); y finalmente, la próxima marca a comprar (bonding). El instrumento de recolección de la información se presenta a continuación.

Instrumentos de entrada y de salida

1. ¿Qué marcas de XXX recuerda? (Escriba cada una de las que recuerde)

2. ¿Recuerda haber visto o escuchado publicidad para algunas marcas de XXX en las 2 últimas semanas? (Escriba cada una de las marcas que recuerda haber visto u oído publicidad)

3. ¿Qué otras marcas de las que aparecen en la imagen adjunta, conoce? (Ver tarjeta de ayuda)

4. De las marcas en la imagen adjunta, ¿para cuáles recuerda haber visto u oído publicidad en las últimas 2 semanas?

5. ¿Cuál es su marca favorita de XXX? (una sola respuesta)

6. La próxima vez que necesite un XXX ¿qué marca comprará? (una sola respuesta)

Nota: Este instrumento se aplicó tanto al inicio como al final de las sesiones. Donde aparecen las equis $(X X X)$ se incluye la categoría del producto.

En el intermedio de la jornada académica, los jóvenes fueron expuestos a mensajes publicitarios estructurados de la siguiente manera:

Para el grupo "televisión" se presentó un video de 7 minutos, que contenía un reel de 8 comerciales donde se incluyeron tres categorías de productos, dentro del cual, en la cuarta posición, se insertó el comercial de la marca Bonfiest para evitar que su ubicación al inicio o al final afectara los resultados. En el grupo "radio", los jóvenes fueron expuestos a una grabación con una canción de moda y un corte comercial con 8 cuñas, ubicando la marca Bonfiest, nuevamente, y por las mismas razones, en la cuarta posición. Para el grupo "revistas", se entregaron ejemplares que contenían la pauta de la marca, al igual que de marcas relativas a las otras dos categorías de productos.

Al finalizar la jornada se aplicó la prueba de salida que midió las mismas variables que la prueba de entrada, para así analizar los cambios presentados en ellas. 
El estudio planteó tres hipótesis, la primera se apoya en la fortaleza de la televisión, pues según Childers y Heckler (1986) es el elemento visual que ha demostrado un efecto más favorable sobre el recuerdo, ya que otorga mayor accesibilidad en la memoria del consumidor. Por tanto, se sugiere la siguiente hipótesis:

H1 Al utilizar solo el medio de comunicación televisión para focalizar la inversión publicitaria, este es capaz de generar crecimiento en todas las evaluaciones de las variables estudiadas.

La segunda hipótesis considera la combinación de diferentes medios de comunicación. El estudio de Chang y Thorson (2004) demuestra que la exposición a diferentes estímulos publicitarios tiene la capacidad de producir efectos mucho más favorables en el consumidor, comparado con una exposición repetida a un mismo estímulo. En línea con esto, se ha formulado la siguiente hipótesis:

H2 Al utilizar la combinación de dos medios convencionales se genera un mayor crecimiento en las evaluaciones de las variables estudiadas, en comparación con el uso de un solo medio.

Por último, la tercera hipótesis se soporta en que los consumidores procesan y codifican la información en su memoria mediante diversas rutas, esta diversidad en la codificación se presenta puesto que cada una de ellas posee diferentes modos sensoriales de percepción. Estas rutas de codificación facilitan la posibilidad de recuperar la información en el momento en que el individuo la necesite para realizar su elección de marca (Appleton-Knapp, Bjork y Wickens, 2005; Sahni, 2015). Por tanto, se sugiere la siguiente proposición:

H3 Al integrar los tres medios de comunicación masivos convencionales se genera el mejor desempeño en los consumidores de las variables estudiadas, especialmente en el vínculo emocional.

\section{RESULTADOS Y DISCUSIÓN}

Luego de realizar las pruebas de entrada y de salida junto con los análisis de las variaciones, se pudo evidenciar un proceso de cambios en el grado de influencia que los diversos medios de comunicación evaluados ejercen sobre las variables analizadas. A continuación, las tablas 1, 2 y 3 presentan los resultados de las variaciones de cada medio de forma independiente. Seguidamente, en las tablas 4, 5 y 6 se exponen los resultados de la exposición a la combinación de diferentes medios de comunicación.

Al utilizar exclusivamente la televisión en una campaña publicitaria, en un proceso comparativo entre la prueba de entrada y la prueba de salida, se observa que la mayoría de los indicadores presentan un buen desempeño. Pese a que la tabla 1 refleja una variación significativa en el desempeño que alcanzó el $60 \%$, también se evidencia que el uso de este medio de manera independiente no tiene la fuerza suficiente para mover el vínculo emocional, pues su indicador generó un decrecimiento del $14 \%$. Por ello se rechaza la primera hipótesis planteada, la cual se basó en el uso exclusivo de la televisión para generar un crecimiento en todas las variables que generan la fortaleza de marca. 
No obstante, se debe reconocer que, al utilizar la televisión como medio exclusivo de inversión publicitaria, es el único medio con el que se logra el efecto más favorable sobre la memoria puesto que eleva al $100 \%$ los resultados en la prueba de salida de las variables Presencia y Relevancia.

\begin{tabular}{|cccc|}
\hline Tabla 1. Resultado del grupo expuesto a publicidad en televisión. & \\
\hline Variables de evaluación & Entrada & Salida & Variaciones \\
\hline Vínculo emocional & $75 \%$ & $66.67 \%$ & $-14 \%$ \\
\hline Ventaja & $58.33 \%$ & $75 \%$ & $29 \%$ \\
\hline Desempeño & $41.67 \%$ & $66.67 \%$ & $60 \%$ \\
\hline Relevancia & $83.33 \%$ & $100 \%$ & $20 \%$ \\
\hline Presencia & $83.33 \%$ & $100 \%$ & $20 \%$ \\
\hline
\end{tabular}

Fuente: Elaboración propia.

Al revisar los resultados del comportamiento de las variables a través de la exposición de los consumidores a la radio, tal como se presenta en la tabla 2, se percibió un crecimiento importante en las cinco variables analizadas. Allí se observan las variaciones positivas en cada una de ellas, donde la más beneficiada fue la variable desempeño, que logró elevar el vínculo en un $25 \%$, frente a la intención inicial para la próxima compra.

Lo anterior demuestra que, para el segmento estudiado, la radio como medio convencional, es capaz de generar crecimientos en todas las variables necesarias para la fortaleza de marca, debido en parte, a que su programación juvenil se ha reinventado logrando estar más conectada con los consumidores.

\begin{tabular}{|cccc|} 
Tabla 2. Resultado del grupo expuesto a publicidad en radio. & \\
\hline Variables de evaluación & Entrada & Salida & Variaciones \\
\hline Vínculo emocional & $28.57 \%$ & $41.67 \%$ & $25 \%$ \\
\hline Ventaja & $28.57 \%$ & $66.67 \%$ & $50 \%$ \\
\hline Desempeño & $50 \%$ & $66.67 \%$ & $71 \%$ \\
\hline Relevancia & $57.14 \%$ & $66.67 \%$ & $50 \%$ \\
\hline Presencia & $71.43 \%$ & $100 \%$ & $40 \%$ \\
\hline
\end{tabular}

Fuente: Elaboración propia.

El efecto de la comunicación exclusiva mediante revistas presentado en la tabla 3 tuvo un resultado poco eficiente en términos construcción de fortaleza de marca, puesto que generó reducciones en la prueba de salida para el $80 \%$ de las variables estudiadas, manteniendo estable solo la variable presencia. Esto demuestra su poca eficiencia al utilizarlo de forma solitaria ya que no genera ningún tipo de crecimiento en las variables.

Tabla 3. Resultado del grupo expuesto a publicidad en revistas

\begin{tabular}{|cccc|}
\hline Variables de evaluación & Entrada & Salida & Variaciones \\
\hline Vínculo emocional & $75 \%$ & $41.67 \%$ & $-44 \%$ \\
\hline Ventaja & $75 \%$ & $66.67 \%$ & $-11 \%$ \\
\hline Desempeño & $75 \%$ & $66.67 \%$ & $-11 \%$ \\
\hline Relevancia & $91.7 \%$ & $66.67 \%$ & $-27 \%$ \\
\hline Presencia & $100 \%$ & $100 \%$ & $0 \%$ \\
\hline
\end{tabular}

Fuente: Elaboración propia. 
Pasando a las combinaciones de medios de comunicación, aunque la mezcla de televisión y radio muestra una mejora considerable en el desempeño del grupo objeto de estudio, su mejor desempeño se concentró en los indicadores de salida de la base de la pirámide (presencia y relevancia), sin lograr movilizar positivamente el vínculo. La tabla 4 demuestra que en este indicador la reducción alcanzó un $33 \%$ frente al resultado del vínculo emocional en la prueba de inicio.

Tabla 4. Resultado grupo expuesto a publicidad en televisión y radio.

\begin{tabular}{|cccc|}
\hline Variables de evaluación & Entrada & Salida & Variaciones \\
\hline Vínculo emocional & $27.27 \%$ & $18.18 \%$ & $-33 \%$ \\
\hline Ventaja & $27.27 \%$ & $27.27 \%$ & $0 \%$ \\
\hline Desempeño & $18.18 \%$ & $45.45 \%$ & $150 \%$ \\
\hline Relevancia & $63.64 \%$ & $81.82 \%$ & $29 \%$ \\
\hline Presencia & $81.82 \%$ & $90.91 \%$ & $11 \%$ \\
\hline
\end{tabular}

Fuente: Elaboración propia.

La combinación de medios televisión y revistas expuesta en la tabla 5, generó incrementos en la mayoría de las variables, aunque el análisis del vínculo mostró una variación negativa del $50 \%$; este resultado es consistente con la evaluación de dos medios (televisión y revistas) de manea independiente, en los cuales también se generó una variación negativa del $14 \%$ y el $44 \%$, respectivamente.

Tabla 5. Resultado grupo expuesto a publicidad en televisión y revistas.

\begin{tabular}{|cccc|}
\hline Variables de evaluación & Entrada & Salida & Variaciones \\
\hline Vínculo emocional & $66.67 \%$ & $33.33 \%$ & $-50 \%$ \\
\hline Ventaja & $66.67 \%$ & $75 \%$ & $13 \%$ \\
\hline Desempeño & $41.67 \%$ & $66.67 \%$ & $60 \%$ \\
\hline Relevancia & $83.33 \%$ & $91.67 \%$ & $10 \%$ \\
\hline Presencia & $91.67 \%$ & $100 \%$ & $9 \%$ \\
\hline
\end{tabular}

Fuente: Elaboración propia.

Por ello frente a $\mathrm{H} 2$, se rechaza el planteamiento en cuanto a que el uso de dos medios convencionales como receptores de inversión publicitaria genera crecimientos en todas las variables de fortaleza de marca.

Por último, la tabla 6 presenta la combinación de los tres medios masivos como son televisión, radio y revistas. De ella se desprende que resulta favorable la inversión publicitaria en esta combinación, puesto que ayuda al crecimiento del $80 \%$ de las variables que construyen fortaleza de marca, es decir incrementa las mediciones de: presencia, relevancia, desempeño y vínculo emocional. Asimismo, mantiene estable la medición en la ventaja.

Tabla 6. Resultado grupo expuesto a publicidad en televisión, radio y revistas.

\begin{tabular}{|c|ccc|}
\hline Variables de evaluación & Entrada & Salida & Variaciones \\
\hline Vínculo emocional & $58.33 \%$ & $83.33 \%$ & $43 \%$ \\
\hline Ventaja & $66.67 \%$ & $66.67 \%$ & $0 \%$ \\
\hline Desempeño & $41.67 \%$ & $75 \%$ & $80 \%$ \\
\hline Relevancia & $83.33 \%$ & $100 \%$ & $20 \%$ \\
\hline Presencia & $91.67 \%$ & $100 \%$ & $9 \%$ \\
\hline
\end{tabular}

Fuente: Elaboración propia. 
En el gráfico 1 puede observarse cómo la triple combinación de medios es la manera más eficiente para elevar la variable vínculo emocional sobre el uso de otras combinaciones o sobre medios utilizados de forma separada.

Los resultados del uso de esta combinación para destinar los recursos de inversión publicitaria son superados por el uso en solitario del medio radio, que mejora los 5 indicadores, en detalle supera al uso de la combinación de medios en 3 de los 5 indicadores evaluados (presencia, relevancia y ventaja).

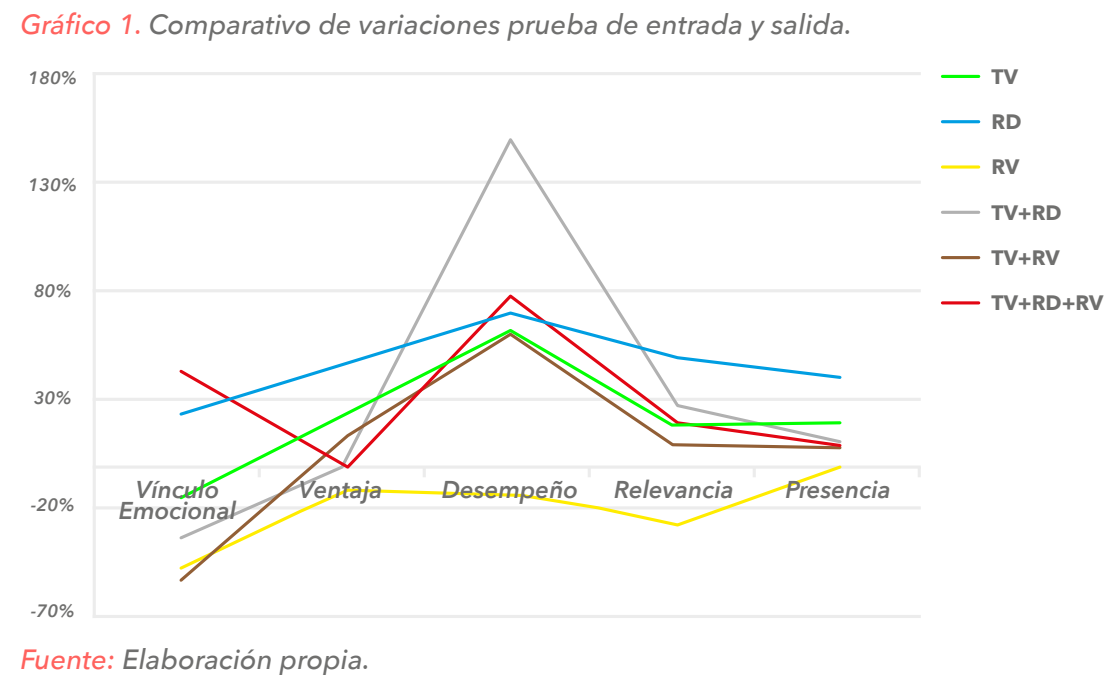

Dentro de este contexto y frente al planteamiento de H3, esta es aceptada parcialmente, pues la combinación de los tres medios de comunicación masivos convencionales a saber: televisión, radio y revistas no es la que mejor desempeño presenta en conjunto. Pero sí es la mejor opción para elevar el desempeño en los resultados de la variable vínculo emocional.

Bajo esta perspectiva, se identificó que sí existe un mayor vínculo del individuo para procesar información cuando se presentó un mismo mensaje a través de diferentes herramientas comunicacionales, convirtiendo su uso en un ejercicio eficiente. Este resultado es concordante con los hallazgos de Stammerjohan, Wood, Chang, y Thorson (2005), al considerar que el uso de herramientas de comunicación múltiple debe ser eficiente, en lugar de extravagante o redundante.

\section{Conclusiones}

El aporte de la comunicación publicitaria es importante en el proceso de fortalecimiento de la marca, sin embargo, los resultados en el mercado dependen de una combinación de factores asociados al producto y a la estrategia global de mercadeo de cada empresa. Esto conlleva en primera instancia a fortalecer la base de la pirámide planteada por la consultora Millward Brown, en términos de presencia y relevancia; estas variables son fundamentales en los procesos de construcción de marca para fortalecerla y crear un posicionamiento en el largo plazo. En la etapa inicial se requiere de un gran esfuerzo en materia de inversión publicitaria, pues los datos analizados permiten afirmar que es mejor el uso de la televisión para elevar los resultados de estas variables. 
Durante una segunda etapa la empresa puede reducir sus presupuestos publicitarios. En ella es conveniente utilizar la radio como medio publicitario para comunicar los reforzadores de las características de sus productos. En el caso concreto de Sal Bonfiest, indicado para aliviar los síntomas asociados al exceso en el consumo de bebidas alcohólicas, como el dolor de cabeza y el malestar estomacal, puede mejorar las variables de desempeño y ventaja.

La combinación de los tres medios masivos tradicionales mejora la última variable del modelo BrandZ, el vínculo emocional de los consumidores jóvenes con la marca, pues su uso permite generar contenidos apropiados para este grupo etario. Esto sugiere la necesidad de replantear la forma de trasmitir el mensaje del producto, lo que podría contribuir al fortalecimiento de la marca.

Finalmente, se recomienda que según los indicadores de crecimiento en la penetración de internet móvil, el uso de los medios digitales puede ser involucrado en la combinación de los medios de comunicación para mejorar la masificación de la información publicitaria.

\section{Referencias}

Appleton-Knall, S. I., Bjork, R. y. Wickens, T. D. (2005). Examining the spacing effect in advertising: encoding variability, retrieval processes, and their interaction. Journal of Consumer Research, 32 (2), 266-276. Recuperado de

http://dx.doi.org/10.1086/432236

Bernal, A. I. (2010). Definición conceptual de los medios de comunicación por un grupo de jóvenes españoles. El valor de Internet. Vivat Academia, (112). Recuperado de

http://pendientedemigracion.ucm.es/inf o/vivataca/numeros/n112/PDFs/AnalBer cop.pdf

Berger, S., Wagner, U. y Schwand, C. (2012). Assessing advertising effectiveness: the potential of goal-directed behavior. Psychology \& Marketing, 29(6), 411-421.

Brandz. (2013). Brand Dynamic Pyramid. Recuperado de http://www.brandz.com/output/Branddy namicpyramid.aspx

Calderón, I. (2014). Jóvenes y medios de comunicación: el desafío de tener que entenderse. Madrid, España: Centro Reina Sofía sobre Adolescencia y Juventud Fundación de Ayuda contra la Drogadicción (FAD)
Cardona Bedoya, J., Riaño Cuevas, D., \& Vaca González, Y. (2017). MARKETING Y CONSUMIDOR GREEN. Aplicación de la Matriz Mic Mac para el análisis de tendencias. LUCIÉRNAGA, 9(17), 12-23. doi: https://bit.ly/34hkGwo

Chang, Y. y Thorson, E. (2004). TV and web advertising synergies. Journal of advertising, (33), 75-84.

Chesnes, M. y Jin, G. Z. (2019). Direct-to-consumer advertising and online search. Information Economics and Policy, 46, 1-22.

Childers, T. y Heckler, S. (1986). Memory for the visual and verbal components of print advertisements. Psychology and Marketing, (13), 137-150.

Cifuentes, C. M. y Sánchez, J. (2006). Condicionamiento clásico de tres tipos de humor en publicidad. Universitas Psychologica, 5(1), 101-126.

Cortés, S., Marván, L. y Lama, C. (2004). Análisis de la publicidad de productos relacionados con la menstruación en revistas dirigidas a adolescentes. Psicología y Salud, 14(001), 113-120. Recuperado de https://bit.ly/3|4N8rk 
Degrado, M. D. (2005). Televisión, publicidad y comunicación. Comunicar, 25, 1-9.

Departamento Administrativo Nacional de Estadística. (2014). Preguntas frecuentes. Recuperado de https://bit.ly/31179is

Departamento Administrativo Nacional de Estadística. (2018). Encuesta de consumo cultural (ECC). Recuperado de https://www.dane.gov.co/index.php/est adisticas-por-tema/cultura/consumo-cult ural

Fischer, L. y Espejo, J. (2011). Mercadotecnia. México: Me Graw Hill.

García, G. F. (2003). Los medios de comunicación, ¿al servicio de la comunidad? En AGUADED, J. I. (Dir.): Luces en el laberinto audiovisual. Huelva: Grupo Comunicar, Agor@ Digital y Universidad de Huelva.

Golovina, N. (2014). La comunicación masiva y el comportamiento del consumidor. Orbis. Revista Científica Ciencias Humanas, 10(28), 190-198.

Keller, K. L. (2003). Strategic Brand Management: Building, Measuring and Managing Brand Equity. Upper Saddle River: Prentice Hall.

Kilian, K. (2009). From Brand Identity to Audio Branding. In Audio Branding. Brands, Sound and Communication. Baden-Baden: Nomos Verlagsgesellschaft / Edition Reinhard Fischer.

Lakshmi, S. y Kavida, V. (2018). Factors contributing to brand positioning of smartphones among college students in Chennai: a study. Journal of Brand Management, 15(19), 55-65.

López, C. D., Sandoval, E. M. y Cortés, P. O. F. (2010). Relación entre los niveles de TRP's, las medidas de recordación, preferencia de marca y la conducta de compra en consumidores colombianos. Psicología desde el Caribe, (25), 30-55.

Marín, D. V. (2006). Medios de comunicación, educación y realidad. Comunicar, (26), 193-197.

Menéndez, T. (2014). La presentación de los jóvenes en los medios de comunicación. Prensa, radio y televisión. Jóvenes y medios de comunicación: e desafío de tener que entenderse. Recuperado de http://adolescenciayjuventud.org
Organización de las Naciones Unidas para la Educación, la Ciencia y la Cultura (1986). La comunicación de masas y la industria publicitaria. Paris: UNESCO.

Otero Gómez, M., Giraldo Pérez, W., \& Vega Barbosa, J. (2018). Comunicación y Mercadeo. Estudio cualitativo para el desarrollo de la marca de Pan de arroz. Luciérnaga, 9(18). doi: https://bit.ly/205GjHH

Ramírez, J. (2001). El paraíso terrestre de la publicidad. Comunicación, 11(3), 1-16. Recuperado de

http://www.repositorio.una.ac.cr/bitstrea m/handle/11056/2106/recurso_203.pdf ?sequence $=1$

Richards, J. I., y Curran, C. M. (2002). Oracles on "advertising": Searching for a definition. Journal of Advertising, 31(2), 63-77.

Román, P. M. (2000). Aspectos metodológicos de la historia de la comunicación. Ámbitos, (5), 119-128.

Rundle-Thiele, S. y Bennett, R. (2001). A brand for all seasons? A discussion of brand loyalty approaches and their applicability for different markets. Journal of Product and Brand Management, 10(1), 25.

Sahni, N. S. (2015). Effect of temporal spacing between advertising exposures: Evidence from online field experiments. Quantitative Marketing and Economics, 13(3), 203-247.

Sanna, D. (2013). Comunicación rentable en marketing. Seis pasos en la era de las redes sociales. Buenos Aires: MarCom Ediciones.

Schultz, D. E. y Kitchen, P. J. (2000). Communicating Globally: An integrated marketing approach. NTC Business Books, Chicago and Macmillan Business, Basingstoke.

Segura, C. A. (2003). Diseños cuasiexperimentales. Facultad Nacional de Salud Pública. Universidad de Antioquia. Recuperado de http://www.sld.cu/galerias/pdf/sitios/ren acip/disenos_cuasiexperimentales.pdf

Stammerjohan, C., Wood, C. M., Chang, Y. y Thorson, E. (2005). An empirical investigation of the interaction between publicity, and previous brand attitudes and knowledge. Journal of Advertising, 34(4), 55-67. 
Torres, E. y Muñoz, J. P. (2006). Publicidad exterior: Estudio exploratorio de recordación de marca y motivación de compra. Revista Venezolana de Gerencia, 11(36), 581-594.

Uribe, R., Manzur, E., Hidalgo, P. y Fernández, R. (2008). Estereotipos de género en la publicidad: un análisis de contenido de las revistas chilenas. Academia. Revista Latinoamericana de Administración, (41), 2008, 1-18.

Wang, H. (2017). A brand-based perspective on differentiation of green brand positioning: A network analysis approach. Management Decision, 55(7), 1460-1475.

Wang, G., Zhuoa, L., Li, J., Ren, D. y Zhang, J. (2018). An efficient method of content-targeted online video advertising. Journal of Visual Communication and Image Representation, 50, 40-48.

Wood, L. (2004). Dimensions of brand purchasing behaviour: Consumers in the 18-24 age group. Journal of Consumer Behaviour, 4(1), 18-24.

\section{Notas}

[1] Artículo resultado de investigación desarrollado por el grupo de Investigación Dinámicas de Consumo de la Universidad de los Llanos.

Para citar este artículo:

Otero Gómez, María Cristina; Giraldo Pérez, Wilson y Giraldo Pérez, Ivan Darío (2019). IMPACTO DE LA PUBLICIDAD DE MARCA EN DIFERENTES MEDIOS DE COMUNICACIÓN MASIVA. Revista Luciérnaga Comunicación. Vol. 11, Núm. 21. Pp $156-171$.

DOI: https://doi.org/10.33571/revistaluciernaga.v11n21a8

OJS. http://revistas.elpoli.edu.co/index.php/luc/issue/archive Link. https://www.politecnicojic.edu.co/index.php/revista-luciernaga 